\title{
Spatial Distribution of the Electric Field in Liquid Crystal Dispersions Devices by using a Finite-Element Method
}

J.M.S. Pena ${ }^{a}$," I. Rodríguez ${ }^{a}$, C. Vázquez ${ }^{a}$, I. Pérez $^{a}$, J.M. Otón ${ }^{b}$

${ }^{a}$ Grupo de Displays y Aplicaciones Fotónicas, Departamento de Tecnología Electrónica, Escuela Politécnica Superior, Universidad Carlos III, C) Butarque, 15 Leganés, E28911 Madrid, Spain

${ }^{b}$ Departamento de Tecnologia Fotónica, E.T.S.I. Telecomunicación, Ciudad Universitaria s/n, E28040 Madrid, Spain

Received 5 November 2002; accepted 10 May 2003

\section{Abstract}

The effective electric field in Polymer/Gel-Glass-Dispersed Liquid Crystal (PDLC/GDLC)

films using an approach based on finite-element method (FEM) has been estimated. A

number of assumptions were considered in the model in order to calculate the electric field over the whole nematic droplet volume. Our aim is to predict the behavior of the electrical magnitudes when some manufacturing parameters of PDLC/GDLC devices such as droplet size, film thickness as well as the director orientation are modified. Results fairly agree with empirical tests described elsewhere. The variations on the electric field distribution due to the interference among contiguous droplets have been studied as well. 


\section{Introduction}

Flat panel displays technology and optoelectronic devices include liquid crystals (LCs) as integrating components among many other innovative materials [1]. They have many unique and useful physical and optical properties allowing fabrication of technologically important electrooptic materials $[2,3]$. A number of interesting applications is related to liquid crystal dispersions in either organic (PDLC) or inorganic (GDLC) matrices [2]. These well known heterogeneous composites consist of microdroplets of liquid crystal dispersed in a polymer or silica matrix. These devices are able to modulate light through electrically-controllable scattering effect [4]. In this way, they can be switched by an external electric field between opaque and clear states without polarisers.

The electric field distribution inside the microdroplets and outside (i.e. in the surrounded matrix) determines the electrooptical properties of these liquid crystal dispersions. In principle, the $\mathrm{AC}$ impedance spectroscopy could be a useful technique to determine electrical properties of these devices in a wide range of frequencies [5]. Bode plots obtained by this measurement technique reproduce a portion of the PDLC/GDLC electrical response; several qualitative effects can be derived from such plots [6].

On the other hand, different approaches have been developed in order to calculate the dielectric response of heterogeneous systems like PDLC and GDLC films [7]. In most practical devices, those models fail to accurately describe their whole electrical properties [8]. 
In this work, we present a powerful method to simulate the spatial distribution of the electric field in both media (nematic droplet and solid matrix) taking into account the director configuration, the LC and matrix electric parameters, and the interference between contiguous droplets.

\section{Electric Field Modeling}

The coordinate system used to study the spatial distribution of the electric field in liquid crystal dispersions (either PDLC or GDLC) devices is shown in Fig. 1. We have used as modeling tool the commercial package Maxwell 2D Field Simulator from Ansoft Corporation, which is based on a finite element method (FEM). This powerful method permits complex analysis in many different cases. The most relevant conditions arising in practical applications are described below.

The evaluation of the electric field is based on one of Maxwell's equations [8]:

$$
\nabla \cdot\left[\tilde{\sigma}^{\prime} * \mathbf{E}+\frac{d}{d t}\left(\widetilde{\varepsilon}^{*} * \mathbf{E}\right)\right]=0
$$

where $\nabla$ is the divergence operator $\widetilde{\sigma}^{\prime}$ and $\widetilde{\varepsilon}^{\prime}$ are the second-order tensors of conductivity and dielectric permittivity, and * means the row-column product. The apostrophe indicates a cylindrical coordinate system. 


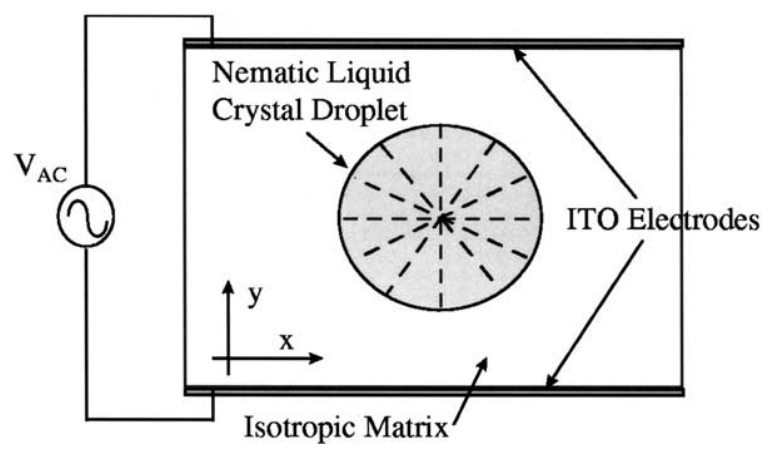

FIGURE 1: The geometry of the problem.

Inside the nematic droplet, using a local principal system of axes for both tensors, and considering that the nematic phase is uniaxial, the following relations may be written:

$$
\begin{gathered}
\widetilde{\sigma}=\left(\begin{array}{cc}
\sigma_{\perp} & 0 \\
0 & \sigma_{11}
\end{array}\right) \\
\widetilde{\varepsilon}=\left(\begin{array}{cc}
\varepsilon_{\perp} & 0 \\
0 & \varepsilon_{11}
\end{array}\right)
\end{gathered}
$$

Let us call $\beta$ the angle between the nematic director and the y-axis of the system. Then,

$$
\widetilde{\sigma}^{\prime}=\sigma_{m}\left(\begin{array}{ll}
1 & 0 \\
0 & 1
\end{array}\right)+\delta \sigma\left(\begin{array}{cc}
-\cos 2 \beta & \sin 2 \beta \\
\sin 2 \beta & \cos 2 \beta
\end{array}\right)=\sigma_{m}+\widetilde{\Sigma}
$$

where the diagonal tensor $\widetilde{\sigma}_{m}$ has been replaced by the scalar $\sigma_{m}$, i.e., the product of $\sigma_{\mathrm{m}}$ by the unit tensor has been omitted for a diagonal tensor with identical diagonal terms describes a scalar property. From this equation, the following relations are derived:

$$
\alpha_{m}=\frac{1}{2}\left(\sigma_{\| 1}+\sigma_{\perp}\right) \quad \text { and } \quad \delta \sigma=\frac{1}{2}\left(\sigma_{\|}-\sigma_{\perp}\right)
$$


Analogous relations can be expressed for the dielectric permittivity:

$$
\widetilde{\varepsilon}^{\prime}=\varepsilon_{m}\left(\begin{array}{ll}
1 & 0 \\
0 & 1
\end{array}\right)+\delta \varepsilon\left(\begin{array}{cc}
-\cos 2 \beta & \sin 2 \beta \\
\sin 2 \beta & \cos 2 \beta
\end{array}\right)=\varepsilon_{m}+\widetilde{\xi}
$$

where

$$
\varepsilon_{m}=\frac{1}{2}\left(\varepsilon_{\|}+\varepsilon_{\perp}\right) \quad \delta \varepsilon=\frac{1}{2}\left(\varepsilon_{\|}-\varepsilon_{\perp}\right)
$$

The electric field inside the nematic droplet arises from several contributions:

$$
\mathbf{E}(\mathrm{t})=\mathbf{E}_{0}(\mathrm{t})+\mathbf{E}_{\mathrm{v1}}(\mathrm{t})+\mathbf{E}_{\mathrm{v} 2}(\mathrm{t})+\mathbf{E}_{\mathrm{s} 1}(\mathrm{t})+\mathbf{E}_{\mathrm{s} 2}(\mathrm{t})
$$

where $\mathbf{E}_{0}$ is the electric field applied along the $y$ axis, with sinusoidal variation with time at an angular frequency $\omega, \mathbf{E}_{v}$ is the field generated by the volume charge density inside the nematic droplet, and $\mathbf{E}_{\mathbf{s}}$ is the field associated to the surface charge density at the dropletmatrix interface.

Using eqs. (4) and (5), eq. (1) may be rewritten as follows:

$$
\nabla \cdot\left[\sigma_{m} \mathbf{E}+\widetilde{\Sigma} * \mathbf{E}+\frac{d}{d t}\left(\varepsilon_{m} \mathbf{E}\right)+\frac{d}{d t}(\widetilde{\xi} * \mathbf{E})\right]=0
$$




\section{Results and Discussion}

A number of assumptions has been made for the simulation of the effective electric field in GDLC films. An average value of the dielectric constant equal to the isotropic phase constant is considered a reasonable approximation of the effective dielectric constant inside the microdroplet [9]

$$
E_{\text {droplet }}=\left(2 \varepsilon_{\perp}+\varepsilon_{\|}\right) / 3
$$

The same approach is used for conductivity,

$$
\sigma_{\text {droplet }}=\left(2 \sigma_{\perp}+\sigma_{\|}\right) / 3
$$

The dielectric constant of any material having non-zero conductivity can be expressed as

$$
\varepsilon^{*}=\varepsilon-\frac{j \sigma}{\omega}
$$

Conductivity is the dominant factor of the complex permittivity in the frequency range where the approximation $\varepsilon \omega / \sigma<<1$ holds. The LC parameters employed in the simulation have been

$$
\begin{array}{ll}
\varepsilon_{\text {droplet }}=11.7 \varepsilon_{0} \mathrm{Fm}^{-1} & \sigma_{\text {droplet }}=9 \cdot 10^{-9} \Omega^{-1} \mathrm{~m}^{-1} \\
\text { droplet radius } a=1 \mu \mathrm{m} & \text { cell thickness } d=40 \mu \mathrm{m}
\end{array}
$$

Figure 2a shows a first case in which the matrix conductivity has been chosen $\sigma_{\text {matrix }}=$ 1.1. $10^{-10} \Omega^{-1} \mathrm{~m}^{-1}$. A remarkable reduction of the electric field magnitude can be observed. In figure $2 b$, the matrix conductivity has been raised up to $\sigma_{\text {matrix }}=1.1 \cdot 10^{-6} \Omega^{-1} \mathrm{~m}^{-1}$. In this case, the field inside the microdroplet is higher than the applied field. This has been 
experimentally confirmed, the effective field inside the LC microdroplet never being higher than 1.5 times the applied field [10]. The convergence error used in either of the above simulation is lower than $1 \times 10^{-5}$.
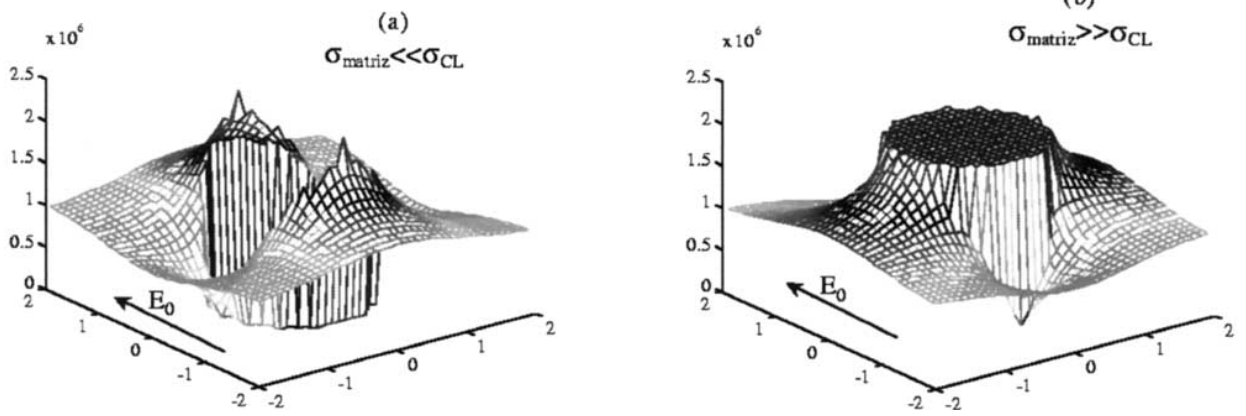

FIGURE 2: Simulated electric field inside a radial nematic droplet for two different cases: (a) when the conductivity of the matrix is much lower than that of nematic droplet and (b) when the conductivity of the matrix is much higher than that of nematic droplet. The height of the lattice represents the relative strength of the electric field. The applied field $\left(\mathrm{E}_{0}\right)$ is along y direction of the local frame.

The above cases assume that the microdroplets are sufficiently far away from each other, so that no interactions between adjacent droplets shall be taken into account. It has been found that for $a / d$ ratios lower than $1 / 3.6$, where $a$ is the droplet radius and $d$ the distance between the centers of the droplets, the spatial electrical interference between adjacent nematic droplets can be negligible [11]. However, this is not usually the actual situation in PDLC and GDLC specimens; hence a realistic approach to these composites must take into account how the electric field inside a microdroplet is affected by its neighbors.

Unfortunately, the problem becomes extremely complex as soon as a number of droplets is placed close to each other. In the following example, an ideal case with two identical droplets at different distances has been considered. The analysed situation employs the following simulating conditions: radius size $a=1 \mu \mathrm{m}$, and the electric field $\mathbf{E}_{0}$ is applied 
along y direction of the local coordinate system. Taking into account the geometry of the problem, similar results are expected if the roles of $x$ and $y$ axes are interchanged.

Spatial electric field simulations along $\mathrm{x}$ and $\mathrm{y}$-directions of the PDLC/GDLC sample have been carried out. Two droplets inside the analyzed volume are considered, and the distance between them has been varied. Results of this analysis for both directions $(\mathrm{x}, \mathrm{y})$ are shown in figures 3,4 . The following values of electrical parameters have been used for the computation:

$$
\sigma_{\text {droplet }} / \sigma_{\text {matrix }}=8.18 \times 10^{-3} ; \varepsilon_{\text {droplet }} / \varepsilon_{\text {matrix }}=0.25
$$

As seen in figure 3 , the electric field inside the nematic droplets remains constant while separation between them is relatively high compared to their radius (same size is taken for both droplets). However, when the distance between droplet centers is reduced, a distortion in the electric field within the nematic spherical cavity is found. The effect becomes highly noticeable when droplets are sufficiently close to each other (i.e., the ratio between the droplet radius and the distance between centers verifies $a / d>>1 / 3.6$ ). For example, it can be seen in figure 4 that the electric field inside the droplets at a relative distance $d=2.2 \mu \mathrm{m}$ becomes more than twice than the field calculated when droplets are far away to each other (i.e., $\mathrm{a} / \mathrm{d}<<1 / 3.6$ ).

A different behavior has been seen along the $y$-direction. In this case, the spatial distribution of the electric field shows a different profile; the variations of the field maximum are negligible.

As expected, the electric field outside the droplet volume also varies when distance is varied (figures 3,4). Nevertheless, its influence on the electrooptical switching of these devices is of secondary importance. 

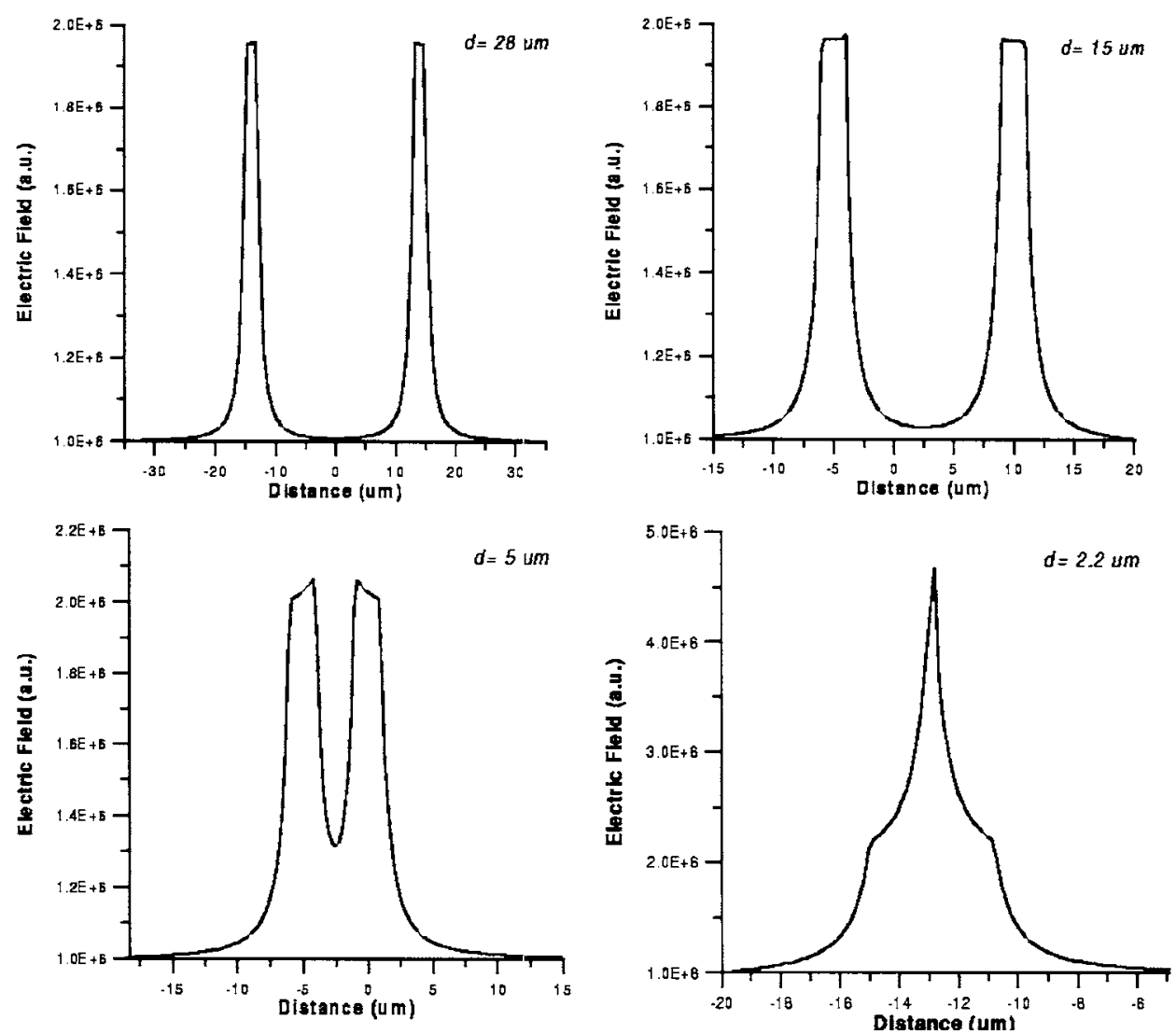

FIGURE 3: Electric field distribution inside two nematic droplets along $\mathrm{x}$-direction for different separation distances (d) between both centers. 

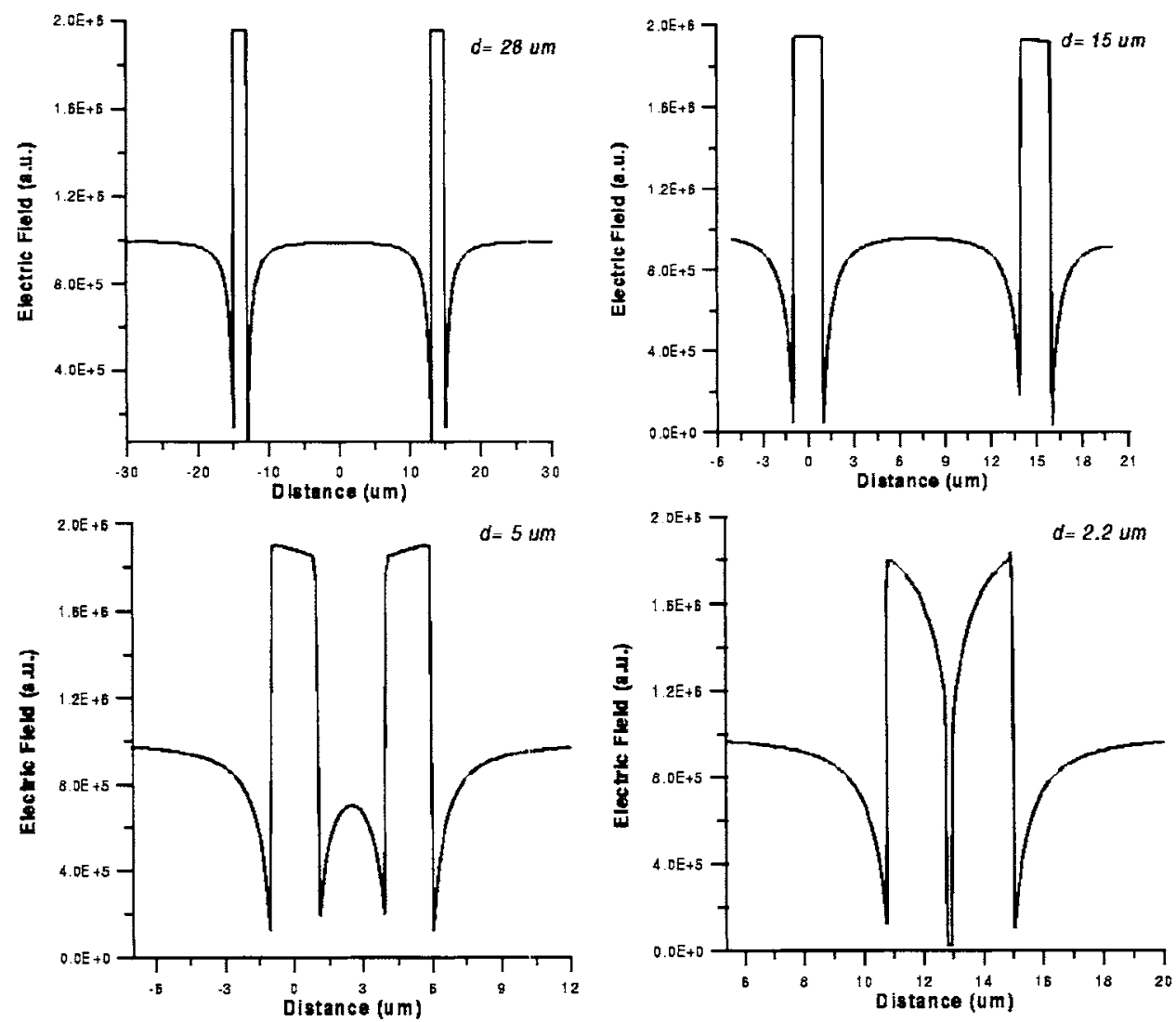

FIGURE 4: Electric field distribution inside two nematic droplets along $y$-direction for different separation distances (d) between both centers.

\section{Conclusions}

An analysis based on FEM simulating the electric field in nematic spherical droplets embedded in an organic or inorganic matrix has been presented. In a simple model considering a single droplet, the electric field inside and outside the droplet for several nematic LC/matrix conductivities has been evaluated.

The model has been eventually extended to the case of two droplets, allowing the study of mutual interferences between them. The electric field distribution along $\mathrm{x}$ and $\mathrm{y}$ directions of PDLC/GDLC films, when an external field is applied along the y direction, shows 
alterations of the magnitude and shape of electric field lines inside and outside the nematic droplets. This effect may affect the reorientation of the director in the whole volume of the nematic spherical cavity producing an anomalous electrooptical switching due to the gradient of the resulting electric field.

FEM reveals to be a successful tool to estimate interactions between adjacent droplets along $\mathrm{x}$ and $\mathrm{y}$ directions. Other directions have not been checked yet. A number of results can be inferred from these simulations in some relevant cases: (i) spatial distribution of the electrical field is chiefly determined by manufacturing parameters such as droplet size, density of droplets per volume unity, droplet separation, as well as electrical properties of both media (liquid crystal and matrix); (ii) moreover, the frequency dependence of the applied field only becomes relevant if the conductivity and dielectric constants are comparable in the liquid crystalline mesophase and the matrix; (iii) the effect of the anisotropy on the electric field distribution within nematic droplets is currently being investigated by the same authors.

\section{Acknowledgements}

This work was supported by CICYT project TIC2000-1594

\section{References}

[1] J.A. Castellano, Handbook of Display Technology, Academic Press, San Diego, 1992.

[2] D. Levy, C.J. Serna, J.M. Otón, Mater. Lett., 10 (1991) 470

[3] L. Petti, P. Mormile, W. J. Blau, Opt. Lasers Eng., (2002) in press,

[4] J.M. Otón, J.M.S. Pena, A. Serrano, Appl. Phys. Lett., 66 (8), (1995) 929

[5] P. S. Drzaic, Liquid Crystal Dispersions, World Scientific, Singapore, 1995.

[6] J.M.S. Pena, E. Olías, X. Quintana, J.M. Otón, Mol. Cryst. Liq. Cryst., 299, (1997) 337

[7] A. Miyamoto, H. Kikuchi, S. Kobayashi, Y. Morimura, T. Kajiyama, Macromolecules, $24,(1991) 3915$

[8] P. Bucci, A. Golemme, J. Chem. Phys. 98 (12) (1995) 10070-10077.

[9] S. Zumer, J.W. Doane, Phys. Rev. A, 34, (1986) 3373

[10] J. Kelly, D. Sekola, Proc. SPIE, 1257, (1990) 17-28.

[11] I. Rodríguez, C. Vázquez, I. Pérez, J.M.S. Pena, Proc. Unión Científica Internacional de Radio (URSI), Vol. I, (1999) 256-257. 